\title{
Regulatory role of nucleophosmin during the differentiation of human liver cancer cells
}

\author{
DONG-HUI XU ${ }^{1 *}$, FAN LIU $^{2 *}$, XIAO LI $^{3}$, XIANG-FENG CHEN ${ }^{3}$, GUANG-JUN JING ${ }^{3}$, \\ FU-YUN WU ${ }^{3}$, SONG-LIN SHI ${ }^{2}$ and QI-FU LI ${ }^{2}$ \\ ${ }^{1}$ Department of Hepatic Biliary Pancreatic Vascular Surgery, the First Affiliated Hospital of Xiamen University, \\ Xiamen, Fujian 361003; ${ }^{2}$ Medical College of Xiamen University; ${ }^{3}$ School of Life Sciences, \\ Xiamen University, Xiamen, Fujian 361102, P.R. China
}

Received January 20, 2014; Accepted March 11, 2014

DOI: $10.3892 /$ ijo.2014.2407

\begin{abstract}
Nucleophosmin (NPM, also known as B23), mainly localized in the nucleolus, has been reported to be overexpressed in many types of human cancer, including colon, ovarian, prostate and gastric cancer. NPM was identified while screening the differential nuclear matrix proteins during HMBA-induced differentiation of human liver cancer cells. We investigated the aberrant expression and subcellular localization of NPM in clinical liver cancer tissues and a cell line with the aim of providing more evidence for revealing the roles of NPM on regulating liver cancer cell proliferation and differentiation. In addition, we studied the potential interaction between NPM and several important proteins. Our results revealed that NPM protein was overexpressed in cancer cells, which was in accordance with the overexpressed mRNA in cancer tissues compared to the corresponding noncancer tissues. We also found a decrease of NPM in protein and mRNA levels upon treatment with the differentiation reagent HMBA. We focused on the aberrant localization of NPM. Immunochemistry and immunofluorescence revealed aberrant cytoplasmic and nucleoplasm localization of NPM in liver cancer tissues and its colocalization with c-Myc, c-Fos, P53 and Rb in the SMMC-7721 cell line. The interactions between NPM and the above proteins were confirmed by GST pull-down assay and co-immunoprecipitation assay. These findings indicate that NPM plays a regulatory role in liver cancer, which deserves in-depth investigation.
\end{abstract}

Correspondence to: Professor Qi-Fu Li or Dr Song-Lin Shi, Medical College of Xiamen University, Xiamen, Fujian 361102, P.R. China

E-mail: chifulee@xmu.edu.cn

E-mail: shisonglin@xmu.edu.cn

*Contributed equally

Key words: nucleophosmin, nuclear matrix, human liver cancer, cell differentiation, protein interaction

\section{Introduction}

Nucleophosmin (NPM) is a ubiquitous nucleolar phosphoprotein with multifunction. NPM has been shown to associate with the assembly and transport of ribosome, the duplication of centrosome and DNA damage response $(1,2)$. NPM regulates the nucleolus activities and cell proliferation, indicating its crucial roles in cell growth. However, the roles of NPM in cell differentiation and the mechanisms of its transportation and relocation are still largely unknown. Recent data suggest NPM is tightly correlated with the development of a tumor (1), its expression level is enhanced in various cancers, such as bladder cancer and prostate cancer (3-5). As a potential proto-oncogene, NPM can affect cell growth through multiple pathways (1). Nevertheless, how NPM affects the reversal of malignant type of tumor cells is still unknown.

In our previous study, we found NPM was a nuclear matrix protein and its expression was downregulated in the induced differentiation of human osteosarcoma, human neuroblastoma tumors and human liver cancer (6-8), indicating NPM functioned in regulating the differentiation of tumor cells (9). Liver cancer is the most common malignant gastrointestinal cancer and the screening for functional proteins related to liver tumor cell differentiation will contribute to the elucidation of the mechanism of liver cancer development and its early diagnosis. Hereby, based on the induced effect of hexamethylene bisacetamide (HMBA) on the differentiation of human liver cancer cells, we extended our study to investigate the location and expression of NPM in the nuclear matrix of human liver cancer cells as well as its relationship with several oncogenes and tumor suppressor genes. Our aim was to provide more evidence to reveal the roles of NPM on regulating liver cancer cell proliferation and differentiation.

\section{Materials and methods}

Ethics statement. The use of human liver cancer tissues in this study was approved by the ethics committees at the Medical College of Xiamen University. Written consent was obtained from each patient with liver cancer. 
Cell line, tissues and other reagents. Human liver cancer SMMC-7721 cells were purchased from China Center for Type Culture Collection. Human liver cancer tissues and matched non-cancerous tissues were obtained from fresh surgical material of patients with liver cancer from the First Affiliated Hospital of Xiamen University (all liver tissues were histologically confirmed). All antibodies used were purchased from Santa Cruz Biotechnology (Santa Cruz, CA, USA) or Cell Signaling Technology, Inc. (CST); the immunohistological kit was purchased from Zhongshan Goldenbridge Biotechnology, Co., Ltd. The PRMI-1640 and bovine serum for cell culture were products of Hyclone (Thermo Scientific). HMBA was purchased from Sigma. RNAiso Plus kit, PrimeScript ${ }^{\mathrm{TM}}$ RT reagent kit and SYBR ${ }^{\circledR}$ Premix Ex Taq ${ }^{\mathrm{TM}}$ were products of Takara.

Cell culture. SMMC-7721 cells were routinely cultured in RPMI-1640 supplemented with $10 \%$ heat-inactivated fetal bovine serum (Hyclone) at $37^{\circ} \mathrm{C}$ in a humidified atmosphere containing 5\% $\mathrm{CO}_{2}$. Twelve hours after seeding, SMMC-7721 cells were maintained in the culture medium containing $5 \mathrm{mmol} / \mathrm{l} \mathrm{HMBA}$ for 7 days to induce differentiation.

Preparation of whole cell lysates and nuclear matrix lysates. The whole cell protein samples were prepared as follows: cells were washed in ice-cold phosphate buffered saline (PBS) and then lysed in lysis buffer [ $7 \mathrm{~mol} / 1$ urea, $2 \mathrm{~mol} / 1$ thiourea, $4 \%$ 3-[(3-cholanidopropyl)dimethylammonio]-1-propanesulfonate (CHAPS), $1.5 \%$ Triton X-100, $1 \%$ pharmalyte (pH 3-10, Bio-Rad), 65 mmol/1 DL-Dithiothreitol (DTT), $40 \mathrm{mmol} / 1$ Tris, complete protease inhibitor cocktail tablets (Roche)]. The suspension was then sonicated for $15 \mathrm{~min}$ at $0^{\circ} \mathrm{C}$ and centrifuged at $12,000 \mathrm{x}$ g for $20 \mathrm{~min}$.

The protein samples of liver tissues were prepared as follows: $0.5 \mathrm{~g}$ of tissue from both cancer and matched normal tissues was frozen in liquid nitrogen and ground with mortar and pestle to yield tissue powder and then suspended in icecold RIPA lysis buffer. The suspension was then sonicated for $15 \mathrm{~min}$ at $0^{\circ} \mathrm{C}$ and centrifuged at $12,000 \mathrm{x}$ g for $20 \mathrm{~min}$.

Nuclear matrix proteins were prepared using a modified method described by Michishita et al (10). After washing in ice-cold PBS twice, SMMC-7721 cells were suspended in cytoskeleton (CSK) buffer $\left[100 \mathrm{mmol} / 1 \mathrm{KCl}, 3 \mathrm{mmol} / 1 \mathrm{MgCl}_{2}\right.$, $5 \mathrm{mmol} / \mathrm{l}$ ethylene glycol tetraacetic acid (EGTA), $10 \mathrm{mmol} / \mathrm{l}$ piperazine-N,N'-bis(2-ethanesulfonic acid) (PIPES), $300 \mathrm{mmol} / \mathrm{l}$ sucrose, $0.5 \%$ Triton X-100, and $2 \mathrm{mmol} / \mathrm{l} \mathrm{PMSF}$, $\mathrm{pH} 6.8$ ] for $10 \mathrm{~min}$ at $0^{\circ} \mathrm{C}$. After being centrifuged at $600 \mathrm{x} \mathrm{g}$ for $5 \mathrm{~min}$, the pellet was resuspended in digestion buffer (identical to CSK buffer except for $50 \mathrm{mmol} / 1 \mathrm{NaCl}$ instead of $\mathrm{KCl}$ ) containing $400 \mu \mathrm{g} / \mathrm{ml} \mathrm{DNaseI}$ for $30 \mathrm{~min}$ at room temperature and centrifuged at $600 \mathrm{x} \mathrm{g}$. Cold ammonium sulfate at a final concentration of $0.25 \mathrm{~mol} / \mathrm{l}$ was used to precipitate proteins. After centrifugation, the pellet was dissolved in lysis buffer [7 mol/1 urea, $2 \mathrm{~mol} / 1$ thiourea, 4\% CHAPS, 1.5\% Triton X-100, 1\% Pharmalyte (pH 3-10 Bio-Rad), 65 mmol/1 DTT, $40 \mathrm{mmol} / 1$ Tris, complete protease inhibitor cocktail tablets (Roche)] and then sonicated at $0^{\circ} \mathrm{C}$ for $20 \mathrm{~min}$. Finally, the suspension was centrifuged at $10,000 \times \mathrm{g}$ and $4^{\circ} \mathrm{C}$ for $30 \mathrm{~min}$ and the supernatants were used as nuclear matrix extracts. Protein concentrations were determined by Bradford assay.
2-DE, MALDI-TOF-MS analysis and protein identification. 2-D PAGE was performed as follows. Protein lysates were diluted in sample buffer with $2 \%$ Immobiline $^{\mathrm{TM}}$ DryStrip gel (IPG) buffer, pH 3-10, nonlinear (GE Healthcare). The samples were applied to IPG Drystrips (17 cm, pH 3-10, GE Healthcare). After isoelectric focusing, the strips were equilibrated and the second dimensional SDS-polyacrylamide gel electrophoresis was carried out. The triplicate sets of silver-stained gels were scanned using a Umax Power Look III photometer and analyzed with the PD Quest 8.0 software (Bio-Rad). The 2-DE gel images were normalized and compared by matching method. Differentially expressed spots were analyzed.

The spots were cut and digested using modified sequencing grade trypsin (Promega). The digested peptides were eluted and dried in freeze drying equipment. After that, the samples were dissolved with $2 \mu \mathrm{l}$ of matrix solution containing $10 \mathrm{mg} / \mathrm{ml}$ alpha cyano-4-hydroxy cinnamic acid (CHCA, Sigma) and were submitted to Bruker III MALDI-TOF mass spectrometer. The spectra were internally calibrated using the trypsin autolysis products by Flex Analysis software and searched against Swiss-Prot and NCBI database using the Mascot tool from Matrix Science. All the searches were analyzed with a 50-ppm mass tolerance.

Western blotting. For western blot experiments, $20 \mu \mathrm{g}$ cell lysates were loaded and separated on polyacrylamide gels and then transferred to polyvinylidene fluoride (PVDF) membranes (Millipore) according to standard protocol. The membranes were blocked for $1 \mathrm{~h}$ at room temperature in 5\% albumin from bovine serum (BSA). The target proteins were probed with primary antibodies and horseradish peroxidase (HRP)-labeled secondary antibodies (Santa Cruz Biotechnology). $\beta$-actin was used as an indicator for equality of lane loading. Antibody positive bands were visualized using ECL western blot detection reagents (Pierce). Quantification of the immunoreactive bands was performed with Quantity One software.

Real-time quantitative RT-PCR. Total RNA was isolated from the cells and tissues using RNAiso Plus kit (Takara). A twostep reverse transcription-PCR procedure was performed using the PrimeScript RT reagent kit (Takara) following the manual instructions. The resultant cDNA was then quantified by Rotor Gene 6000 with SYBR Premix Ex Taq (Takara). The expression level of NPM was normalized to $\beta$-actin mRNA. The PCR primers used were: i) NPM-F (5'-GGAGGTG GTAGCAAGGTTCC) and NPM-R (5'-TTCACTGGCGCT TTTTCTTCA); ii) $\beta$-actin-F (5'-CATGTACGTTGCTAT CCAGGC) and $\beta$-actin-R (5'-CTCCTTAATGTCACGCAC GAT). All PCR reactions were performed in triplicates to ensure reproducibility.

Immunohistochemistry analysis. Immunohistochemical analyses were performed by the following methods. Sections of $5 \mu \mathrm{m}$ were taken from tissue block and affixed to polylysine coated slides and air-dried overnight at $37^{\circ} \mathrm{C}$. After dewaxing and antigen retrieval, endogenous peroxidase was quenched with $3 \%$ hydrogen peroxide for $10 \mathrm{~min}$. After washing in distilled water three times, the cover slips were blocked for $30 \mathrm{~min}$ with $3 \%$ BSA at room temperature and then incubated in a mixture of primary antibodies (mouse monoclonal anti-NPM, 1:200 


\section{A $\operatorname{IEF}(3-9.5)$}
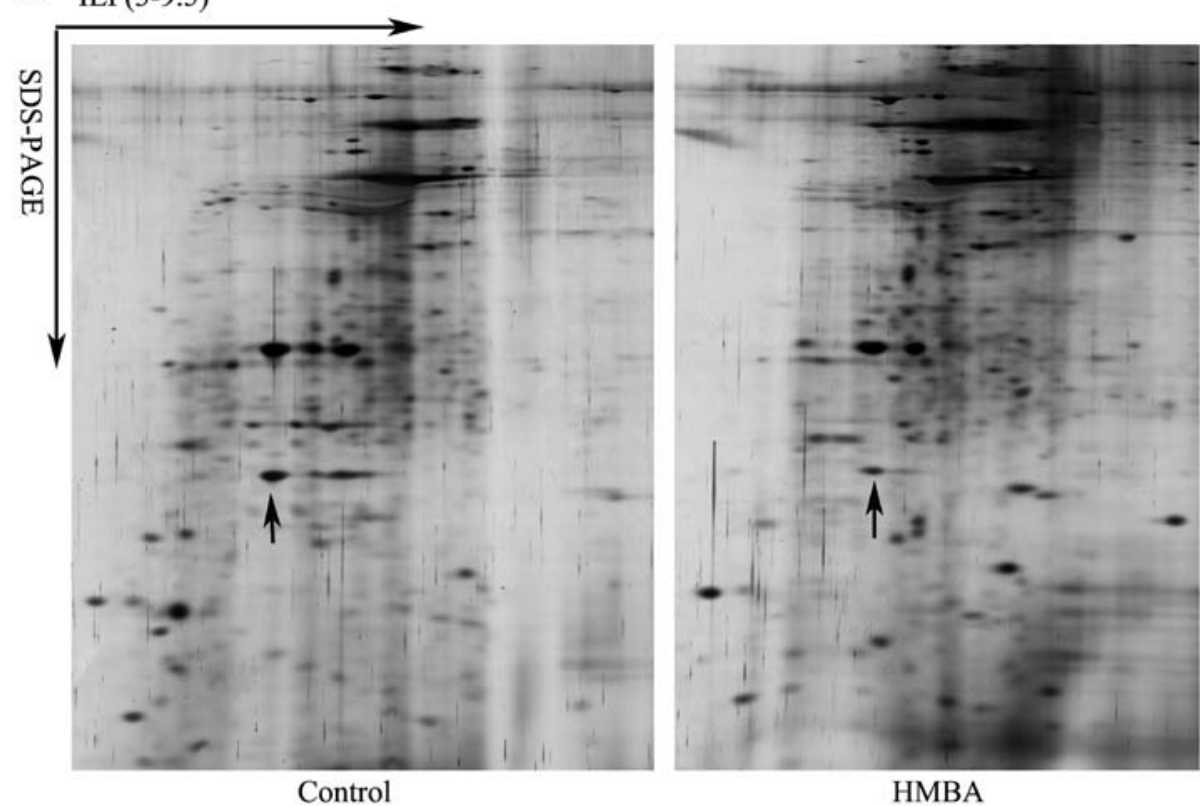

HMBA

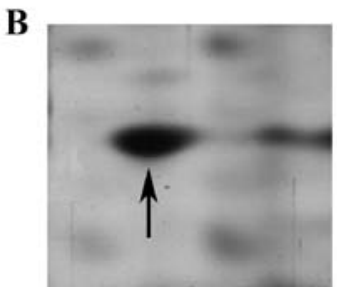

Control

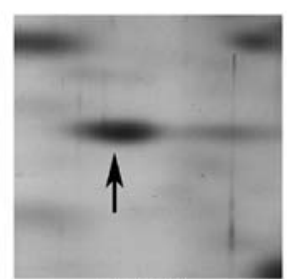

HMBA

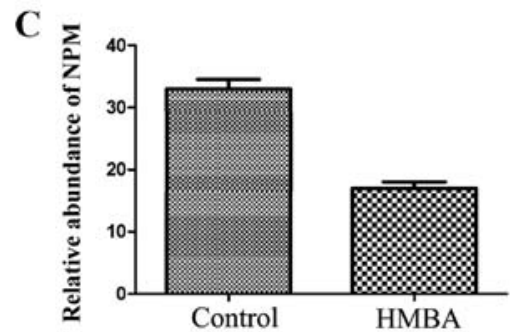

Figure 1. Representative 2-D PAGE profiles of nuclear matrix proteins from SMMC-7721 cells visualized by silver staining. (A) The arrow showed the protein spots corresponding to nucleophosmin in 2-DE PAGE gels of nuclear matrix proteins from SMMC-7721 cells before and after HMBA treatment. (B) Enlarged maps of changed expression of NPM from SMMC-7721 cells. (C) Relative abundance of NPM in nuclear matrix was normalized with PD Quest 8.0 software by comparing the optical density of the MPM spots to total optical density of all the spots in the gel.

dilution, Santa Cruz Biotechnology) diluted in 2\% BSA. After overnight incubation, the cover slips were washed thoroughly with PBST (PBS, 0.05\% Tween-20). The next steps were performed according to the manual of the 2-step plus ${ }^{\circledR}$ polyHRP anti-mouse/rabbit IgG detection system (ZSGB-Bio Co., Ltd.). The antigen-antibody complex was visualized with diaminobenzidine (DAB) substrate. Images of the stained sections (three sections per sample) were captured and the quantitative analysis was performed with Image-Pro Plus 6.0 software.

For the samples of immunofluorescence analysis, the CY3-conjugated goat anti-mouse IgG was used as secondary antibody in 2\% BSA for $1 \mathrm{~h}$ at room temperature in the dark. After three washes in PBS, the sections were sealed with nail polish to prevent movement while observed under a laser confocal scanning microscope.

Laser-scanning confocal microscopy for double-immunofluorescence analysis. Cells grown on cover slips were fixed in $4 \%$ parafomaldehyde for $10 \mathrm{~min}$ at room temperature and then washed in PBS. For permeabilization the cells were immersed in PBS containing 0.5\% Triton X-100 for 10 min and then washed in PBS three times for $5 \mathrm{~min}$. After blocking in $1 \%$ BSA in PBST (PBS containing $0.05 \%$ Tween-20), cells were incubated in a mixture of two primary antibodies (mouse anti-human NPM/rabbit anti-human c-Fos, mouse anti-human NPM/rabbit anti-human c-Myc, mouse antihuman NPM/rabbit anti-human P53, mouse anti-human NPM/ rabbit anti-human $\mathrm{Rb}$ ) in $1 \%$ BSA in PBST in a humidified chamber for $1 \mathrm{~h}$ at room temperature. After washed three times in PBS for 5 min cells were incubated with a mixture of two secondary antibodies (FITC-conjugated goat anti-mouse IgG/CY3-conjugated goat anti-rabbit IgG) in $1 \%$ BSA for $1 \mathrm{~h}$ at room temperature in the dark. After three washes in PBS, the cells were incubated in DAPI for $5 \mathrm{~min}$ in the dark and rinsed in PBS, then mounted with a drop of mounting medium and sealed with nail polish to prevent movement under the microscope. Image acquisition was performed with laser confocal scanning microscopy (TCS-SP2 MP, Leica). For the sections of cancer tissues and adjacent normal tissues, after dewaxing and antigen retrieval, the following steps were in accord with the standard method of cell samples.

Construction of GST-NPM expression vector and GST pulldown assay. Full-length NPM was isolated from SMMC-7721 cells by RT-PCR using NPM-specific primers. The primers used were: NPM-F (5'-CGCGGATCCATGGAAGATTCG ATGGACAT) and NPM-R (5'-CCGCTCGAGAAAGAGAC TTCCTCCACTGCC). The full-length NPM was then sequenced and cloned into the expression vector pGEX-4T-2 
Table I. NPM protein was identified by searching MASCOT database (www.matrixscience.com).

\begin{tabular}{lcccr}
\hline Protein name & NCBI entry & Mol. Mass calc (Da) & pI (calc) & Score \\
\hline Nucleophosmin & gi83641870 & 28497 & 4.56 & 176 \\
\hline
\end{tabular}
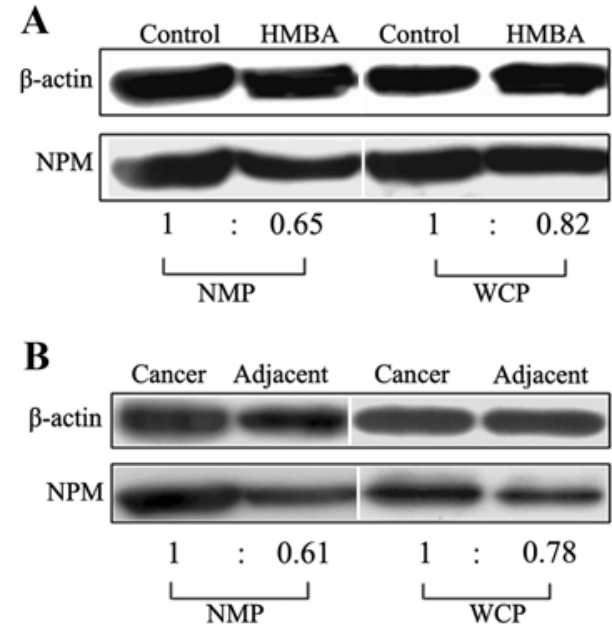

Figure 2. Expression levels of NPM in tumor cells and liver cancer tissues. The nuclear matrix proteins and the whole cell proteins were extracted from SMMC-7721 cells and human liver cancer tissues. (A) The results of western blotting showed that NPM decreased both in nuclear matrix proteins and in whole cell proteins of the HMBA-treated cells. (B) Representative results of NPM expression in cancerous tissues and their adjacent non-cancerous tissues. NMP, nuclear matrix proteins; WCP, whole cell proteins.

with glutathione S-transferase (GST) tag at the $\mathrm{N}$ terminus. The E.coli-expressed GST-fusion (bait) protein, GST-NPM, is immobilized onto the MagneGST Particles (Promega) to affinity purify any proteins (prey) from SMMC-7721 cell lysates according to the technical manual of Promega MagneGST ${ }^{\mathrm{TM}}$ pull-down system. The bait-prey protein complexes contained in eluted samples were further analyzed by SDS-PAGE and western blotting.

Co-immunoprecipitation (Co-IP) assay. A typical experiment used $1000 \mu \mathrm{l}$ of whole cell lysate (WCL) of liver tumor cells (containing $1000 \mu \mathrm{g}$ of soluble protein), and was incubated with $1 \mu \mathrm{g}$ of purified NPM IgG for $4 \mathrm{~h}$ or overnight at $4^{\circ} \mathrm{C}$. Control immunoprecipitation experiments included $1 \mu \mathrm{g}$ of purified pre-immune IgG or irrelevant IgG. WCL (50 $\mu \mathrm{l})$ was used as positive control. A $80-\mu 1$ aliquot of a $50 \%$ (v/v) slurry of protein A-coupled Sepharose beads was then added and incubated for $2 \mathrm{~h}$ at $4^{\circ} \mathrm{C}$. The beads was pelleted in a microcentrifuge $(3,000 \times \mathrm{g}$ for $30 \mathrm{sec})$ and washed three times in wash buffer (identical to lysis buffer). Then the beads were boiled in reducing SDS-PAGE sample loading buffer and analyzed by SDS-PAGE and western blotting.

\section{Results}

Identification of NPM in nuclear matrix lysates of liver cells by MALDI-TOF-MS. The nuclear matrix lysates of SMMC-7721 cells before and after HMBA treatment were subjected to 2-DE PAGE followed by silver-staining of the gel. All procedures (extraction of nuclear matrix proteins, 2-DE PAGE and silverstaining) were independently repeated three times to ensure the reproducibility of 2-DE PAGE. Representative gel images are shown in Fig. 1A. PD Quest 8.0 software (Bio-Rad) was used for quantification analysis. The differential protein spots in intensity were excised and digested with typsin and then were identified by mass spectrometry. After searching against MASCOT database (www.matrixscience.com) the protein spot labeled with an arrow in Fig. 1B was identified as NPM (Table I). Fig. 1B is the enlarged maps of NPM from 2-DE gels and Fig. 1C shows the relative expression level of NPM in cells before and after HMBA treatment.

Upregulation of NPM protein level both in SMMC-7721 cells and liver cancer tissues. To verify the existence and abberant expression of NPM in nulcear matrix and liver tissues, western blot analysis was performed. The whole cell lysates and nuclear matrix lysates of SMMC-7721 cells and human liver tissues and matched normal tissues were separated by SDS-PAGE and then transferred to PVDF membrane in semi-dry conditions according to the standard protocol. The results showed that when compared with that in the differentiated cells the protein level of NPM in liver tumor SMMC-7721 cells was improved both in nuclear matrix and whole cell proteins (Fig. 2A). For uncovering the possible changes of NPM in liver cancer development, we further probed the expression level of NPM in human liver cancer tissues. We found the expression level of NPM in liver cancer tissues was much higher than that in adjacent non-cancerous tissues among 30 patients who were clinically diagnosed with liver cancer (Fig. 2B).

Upregulation of NPM mRNA level both in SMMC-7721 cells and liver cancer tissues. Western blot data suggested that NPM protein was overexpressed in SMMC-7721 cells and liver cancer tissues. To investigate whether the mRNA level of NPM was changed, the mRNA expression of NPM was examined in SMMC-7721 cells with or without HMBAtreatment and in liver cancer tissues and matched normal tissues. Consistent with the protein expression, NPM was highly expressed in SMMC-7721 cells compared with differentiated cells (Fig. 3A). Of the paired liver cancer tissues analyzed, all the cases showed enhanced NPM mRNA expression compared with the matched normal tissues (Fig. 3B-D).

Evaluation of NPM expression in liver cancer tissues. To investigate the expression of NPM in liver cancer tissues, immunohistochemistry and immunofluorescence was performed to detect NPM expression in paired primary liver cancer and their adjacent non-cancer tissues. In addition, results showed that the brown-yellow granules (Fig. 4A and $\mathrm{B}$ ) and the red fluorescence (Fig. 4C and D) of NPM were mainly 
A

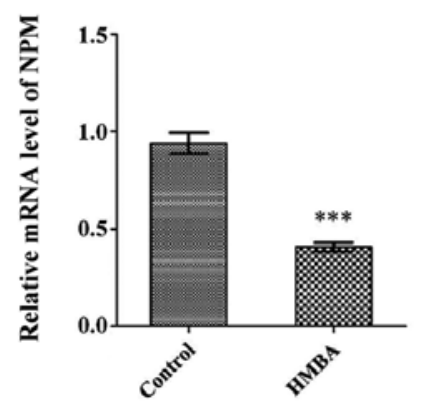

B

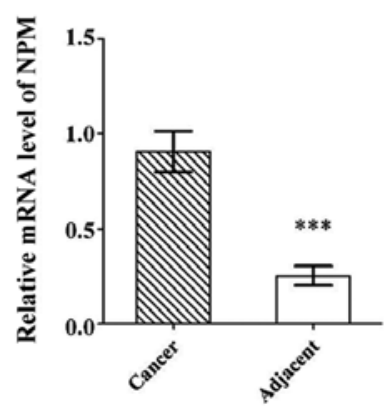

C

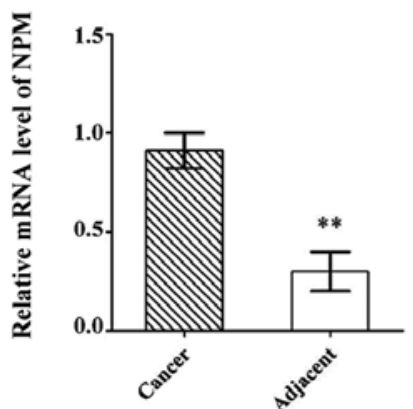

D

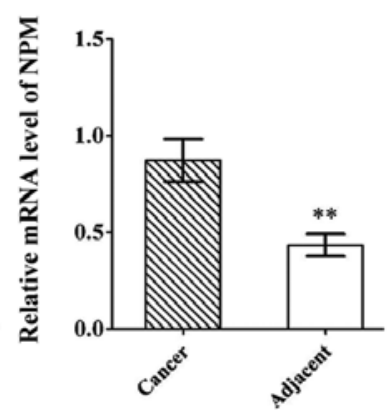

Figure 3. The mRNA expression levels of NPM decreased in human liver cell lines treated by HMBA and non-cancer tissues. The mRNA of NPM in human liver cell lines (A) and primary liver cancer and their non-cancer tissues (B-D) were measured by fluorescent quantitative real-time PCR. The results are expressed as the ratio of copies of NPM relevant to $\beta$-actin from three independent experiments. Data are expressed as mean $\pm \mathrm{SD} ;{ }^{* * *} \mathrm{P}<0.01,{ }^{* * * *} \mathrm{P}<0.001$.
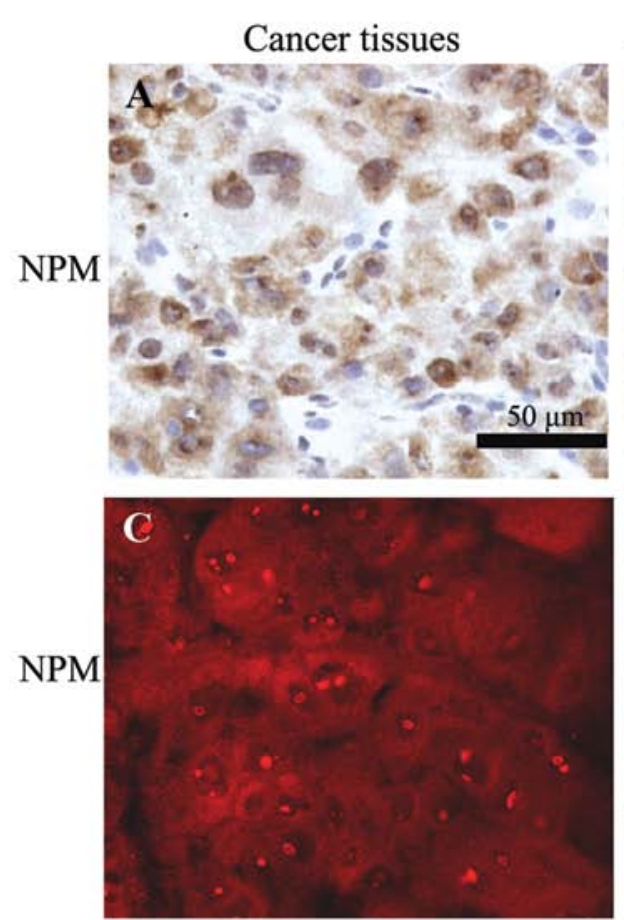

Adjactis
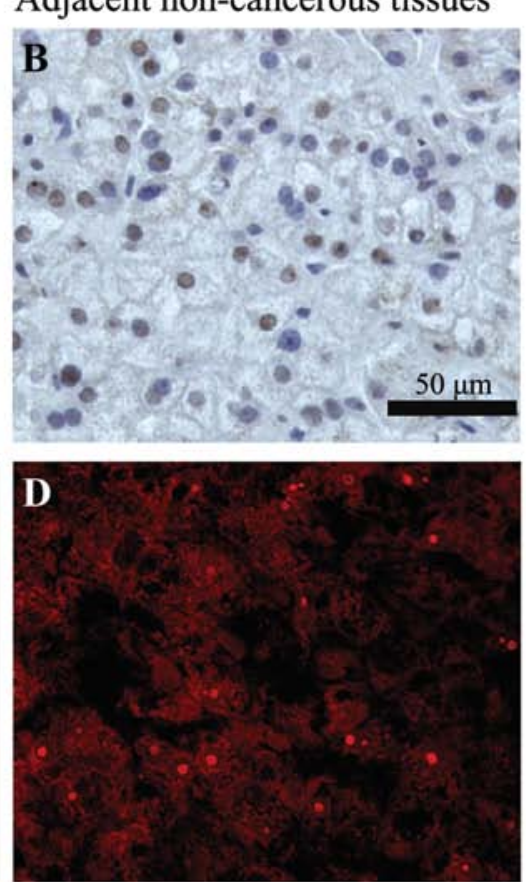

$\mathbf{E}$

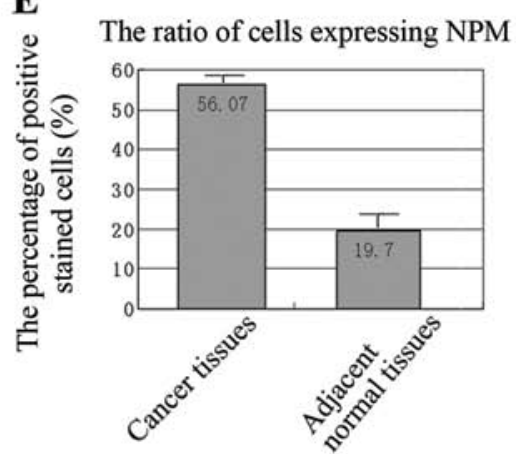

$\mathbf{F}$

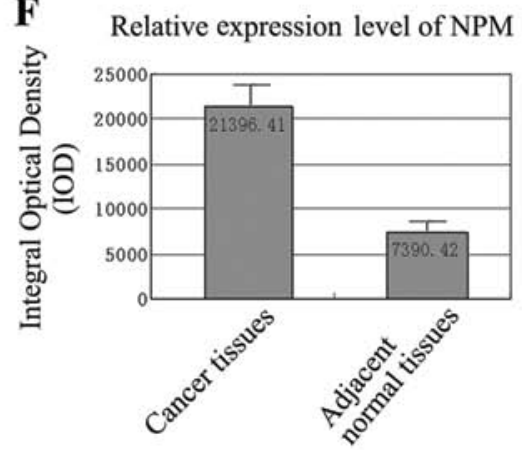

Figure 4. Evaluation of NPM expression in liver cancer tissues using immunohistochemical and immunofluorescence assays. The brown-yellow granules (A) and the red fluorescence (C) of NPM were mainly expressed in the nucleus and the cytoplasm of cancer tissues. NPM was strongly expressed in primary liver cancer tissues whereas weakly expressed in the nucleus of the adjacent non-cancer tissues (B and D). Quantitative analysis of NPM expression with Image-Pro Plus software (E and F) showed that the ratio of positively stained cells altered from $56.07 \%$ in cancer tissues to $19.70 \%$ in adjacent normal tissues and the relative expression level of NPM decreased by $65 \%$.

expressed in the nucleus and the cytoplasm of cancer tissues whereas weakly expressed in the nucleus of adjacent noncancer tissues. Quantitative analysis of NPM expression with Image-Pro Plus software showed that the ratio of positively stained cell altered from $56.07 \%$ in cancer tissues to $19.70 \%$ in adjacent normal tissues and the relative expression level of NPM decreased by $65 \%$ (Fig. 4E and F).

Intracellular colocalization of NPM with c-Fos, $c-M y c, P 53$ and $R b$. To corroborate the physical proximity of NPM with c-Fos, c-Myc, P53 and Rb at the subcellular level, the double immunofluorescent staining method was employed. The green fluorescence representing NPM was observed in the nucleus especially around the periphery of the nucleolus, and some weak fluorescent signal could be detected in the cytoplasm in control SMMC-7721 cells; after HMBA treatment the green fluorescence was mainly assembled in the nucleolus, the intensity of green fluorescence decreased. The yellow fluorescence indicates the colocalization of NPM with other proteins labeled by red $\mathrm{Cy} 3$.

Colocalization of NPM with c-Fos in liver tumor cells. The red fluorescence representing c-Fos accumulated both in nucleus and in the cytoplasm. NPM colocalized with c-Fos in the nucleus, especially around the regions of the nucleolus (Fig. 5A). After HMBA treatment the intensity of yellow fluorescence in nucleus was dramatically weakened and the colocalization is mainly located in the nucleolus (Fig. 5B). 

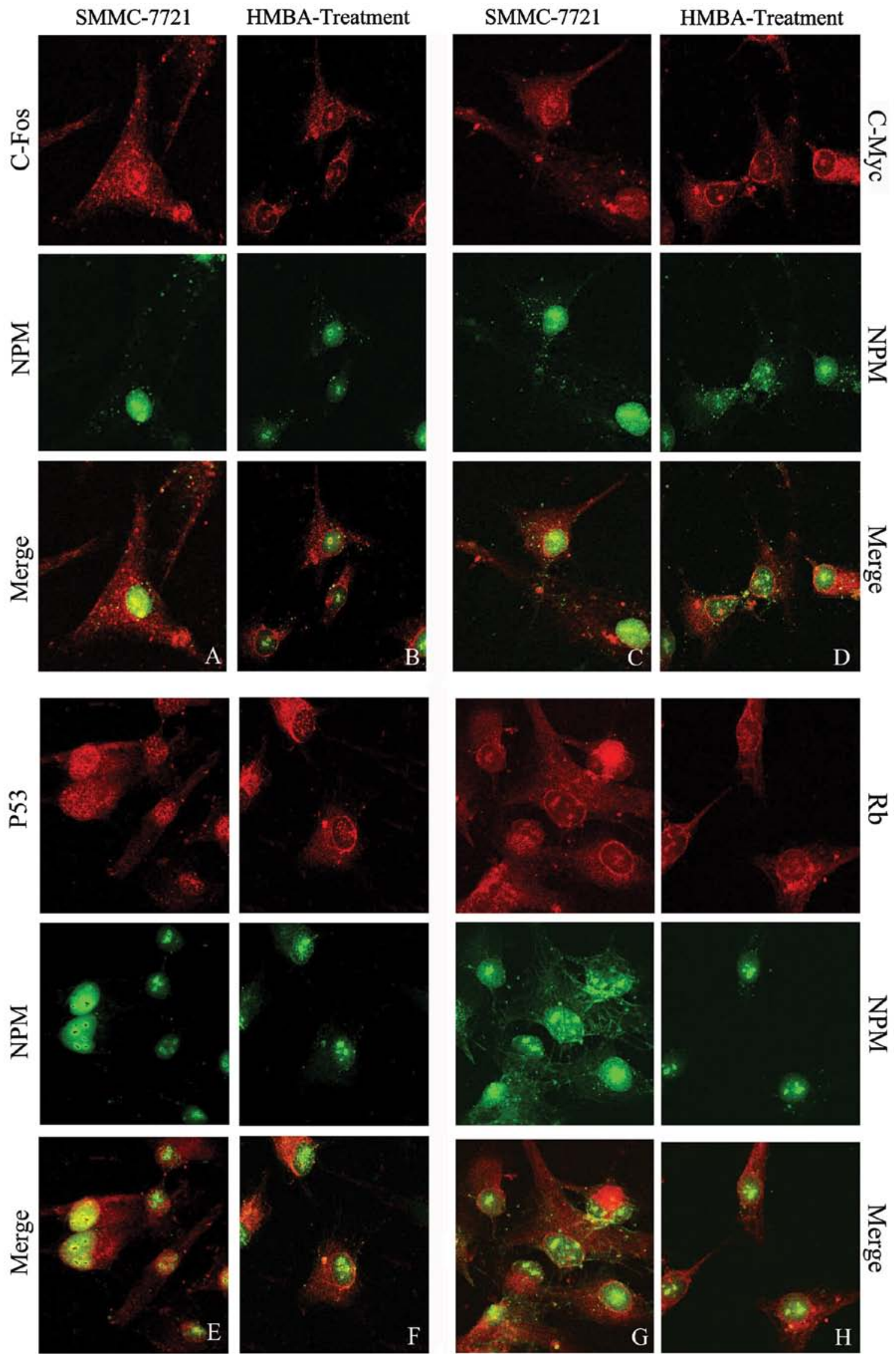

Figure 5. Intracellular colocalization of NPM with c-Fos, c-Myc, P53 and Rb. Double staining of NPM (green) and the expression products of other oncogenes or tumor suppressor genes (red) in SMMC-7721 cells pre- and post-HMBA treatment were performed and the cells were observed by laser-scanning confocal microscopy. The yellow or orange fluorescence indicates the colocalization of NPM with other proteins. The merged image shows the colocalization of NPM with c-Fos in SMMC-7721 cells (A) and HMBA-treated cells (B). The colocalization of NPM with c-Myc in SMMC-7721 cells (C) and HMBA-treated cells (D). The colocalization of NPM with P53 in SMMC-7721 cells (E) and HMBA-treated cells (F). The colocalization of NPM with P53 in SMMC-7721 cells (G) and HMBA-treated cells (H). 
A

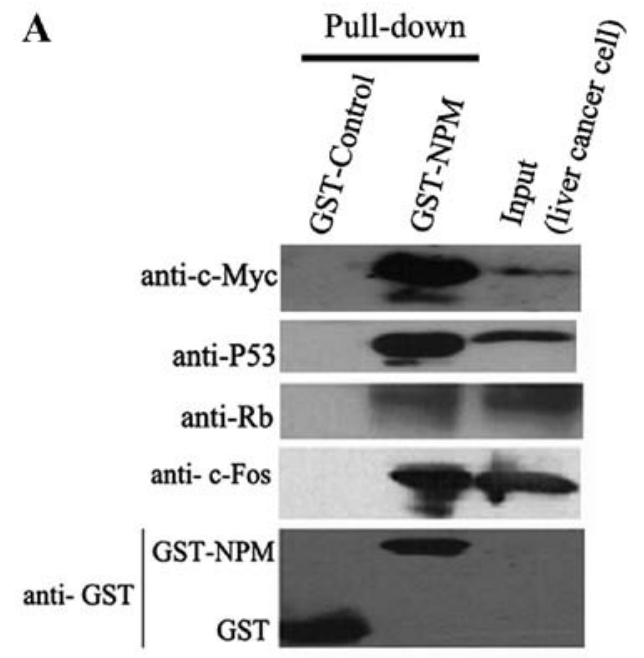

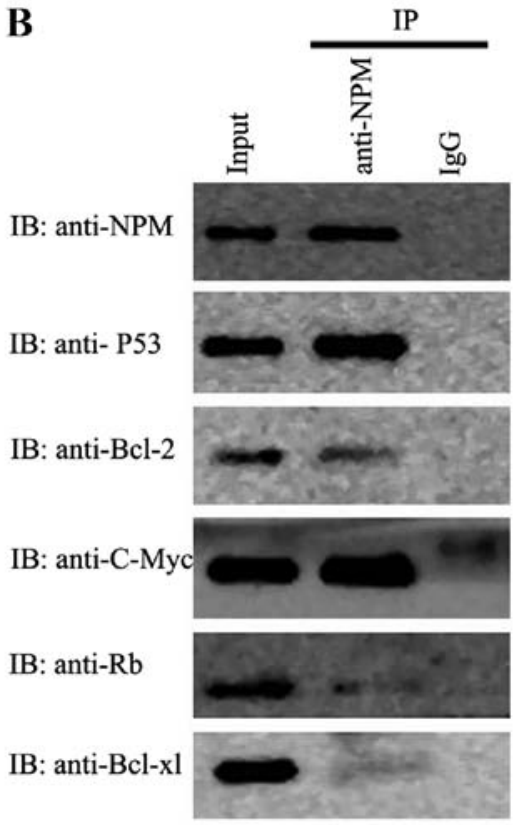

Figure 6. Interaction of NPM with c-Myc, c-Fos, P53 and Rb in liver cancer cells by GST pull-down assay. (A) Pull-down results showed that NPM interacts with c-Myc, c-Fos, P53 and Rb. Protein expressed from pGEX-4T-2 vector was used as negative control and the cell lysates of SMMC-7721 cells were used as positive control. (B) Co-immunoprecipitation of NPM was tested by western blotting. Purified rabbit polyclonal antibody was linked to low leaching protein A-agarose beads and used to immunoprecipitate (IP) NPM complexes from $1000 \mu \mathrm{g}$ of SMMC-7721 whole cell lysate (WCL). The WCL was used as positive control (Input), and irrelevant IgG was used as negative control. The labels on the left of each panel show the antibodies used for analysis of immunoprecipitated material by western blotting.

Colocalization of NPM with c-Myc in liver tumor cells. The observation of laser confocal microscope showed the c-Myc red fluorescence was distributed through the whole cell. The merged picture shows NPM obviously colocalized with c-Myc in the nucleolar periphery (Fig. 5C); HMBA treatment resulted in the decreased fluorescent intensity of c-Myc in the nucleus, and the fluorescent intensity in the cytoplasm was much stronger than that in the nucleus (Fig. 5D). The colocalization of NPM with c-Myc was observed in the cytoplasm and nucleolus in the differentiated cells, which demonstrated a tendency for the colocalization of NPM and c-Myc to transfer from the nucleolus to the cytoplasm.

Colocalization of NPM with P53 in liver tumor cells. In SMMC-7721 cells the red fluorescence representing P53 was distributed both in the nucleus and cytoplasm, but the fluorescent intensity in the nucleus was much stronger than that in the cytoplasm. The merged image reveals that the strong colocalization of NPM with P53 widely distributed in the nucleus especially in the nucleolar periphery (Fig. 5E). In HMBAtreated cells, the merged results show the yellow fluorescence as weak in the nuclear region, indicating the colocalization between P53 and NPM was decreased and transferred outside the nucleus (Fig. 5F).

Colocalization of NPM with Rb in liver tumor cells. The red fluorescence representing $\mathrm{Rb}$ accumulated both in nucleus and cytoplasm (Fig. 5G). After HMBA treatment the Rb red fluorescence dispersed in the cytoplasm, especially around the periphery of the nucleus. The yellow fluorescence showed the colocalization of NPM and Rb mainly distributed in the nucleolus (Fig. 5H).
Interaction of NPM with $c-M y c, c-F o s, P 53, B c l-2, B c l-x l$ and $R b$ in liver cancer cells. To verify the potential interactions indicated by colocalization between NPM and other proteins, GST-NPM fusion protein was constructed and used in a pulldown assay. For western blot analysis, GST protein expressed from pGEX-4T-2 vector was used as negetive control and the cell lysates of SMMC-7721 cells was used as positive control. Pull-down results showed that NPM interacts with c-Myc, c-Fos, P53 and Rb (Fig. 6A). Co-IP assay further verified the interactions between NPM and these proteins in vivo. Moreover, co-IP assay found the interactions between NPM and Bcl-2 or Bcl-xl (Fig. 6B).

\section{Discussion}

Expression changes of NPM in nuclear matrix during the differentiation of liver tumor cells. As a ubiquitous nucleolar phosphoprotein, NPM is involved in a series of biological processes, such as the assembly and transport of ribosome, the duplication of centrosome and the DNA damage response. The location and expression changes of NPM are closely related to cell proliferation and carcinogenesis. In the present study proteomic analysis revealed that NPM was included in the protein components of liver cancer cell nuclear matrix, moreover, its expression level was downregulated after HMBA-induced differentiation, which was further confirmed by western blot assay. Hsu et al (11) reported the downregulation of NPM in RA-induced HL-60 cells, and in our previous study we also found decreased level of NPM during the differentiation of tumor cells (6), which was consistent with the results of the present study. Overexpression of NPM resulted in high cellular growth rate (12). Data in this study further confirmed that NPM was a nuclear matrix protein 
and its expression level was decreased in differentiated tumor cells, proving that NPM played crucial roles on regulating the differentiation of liver cancer cells.

Expression alterations of NPM both in liver cancer cells and liver cancer tissues and matched non-cancer tissues. The aberrant expression of NPM in human cancer tissues has been widely reported. Accumulated data showed the overexpression of NPM in tumor cells (13-15). In this study we found NPM was overexpressed in human liver cancer cells both at protein and at mRNA levels; the immunocytochemical results showed NPM was mainly expressed in the nucleolus and nucleoplasm, and it was concentrated into the nucleolus after HMBA treatment. For further revealing the expression and location of NPM in human liver cancer tissues, we performed immunohistochemistry on the paraffin sections of cancer tissues. The results, for the first time, displayed that NPM expression was strongly positive in the cytoplasm of liver cancer tissues while it was weakly positive in the nucleus of matched non-cancer tissues, indicating differential location and expression level of NPM during human liver carcinogenesis, which corroborated the data from liver cell lines. Development of bladder cancer and liver cancer associated with the enhanced expression of NPM $(4,16)$, and higher NPM protein level resulted in more advanced tumor stages, grades, poor prognosis, and likelihood of recurrence (4), moreover, the mRNA levels of NPM was higher in cancerous tissues when compared with normal tissues (17), and our results further confirmed the enhanced mRNA level of NPM in liver cancer tissues.

In recent years, studies have been carried out on the functional roles of NPM in the differentiation of tumor cells, however, the detailed mechanisms are still unknown. Our present study showed the differential location and expression level of NPM either in liver cell lines or in human liver cancer tissues, indicating its crucial roles in liver carcinogenesis.

Interaction and altered colocalization of NPM with oncogenes and tumor suppressor genes. As an important functional regulatory protein, NPM can regulate the activities of several genes, such as p53 and p14 (18). NPM might regulate the differentiation of tumor cells through interaction with some proteins of oncogenes and tumor suppressor genes.

In the present study, laser confocal scanning microscope revealed NPM colocalized with c-Fos, c-Myc, P53 and Rb at the nucleolar periphery of liver tumor cells; moreover, the colocalized regions were translocated due to the HMBA-induced differentiation. The colocalized relationship indicates the potential interaction of NPM with c-Fos, c-Myc, P53 and Rb.

The oncogene c-myc, a member of myc gene family, is closely related to cell proliferation. The protein of c-myc can directly interact with NPM (19-22). Our results corroborated the deductions and found that NPM colocalized with c-Myc in the periphery of nucleolus. In the differentiated liver cells, NPM coexpressed with c-Myc in the nucleoli and the cytoplasm, indicating the interaction between NPM and c-Myc was a dynamic process. How NPM and c-Myc shuttled from the nucleoli to the cytoplasm needs more consideration. C-Fos can improve the transcriptional activities of several genes related to cell proliferation through binding with c-Jun. We, for the first time, reported that NPM interacted with c-Fos in the nucleolus and nucleoplasm of tumor cells, and the colocalized locus changed during the differentiation of tumor cells. These results indicated NPM may directly or indirectly interact with c-Fos and further regulated cell differentiation. We also report here that NPM interacted with Bcl-2 and Bcl-xl, both of which are apoptosis-related proteins, indicating the potential role of NPM in apoptosis regulation. There is no previous report on the interactions between Bcl-2, Bcl-xl and NPM. It was reported that NPM was an inhibitor of p53, NPM could suppress the activity of p53 in DNA damage response (23). NPM directly interacted with p53 and coexpressed at the nucleolus (24). Our results further corroborated the interactions between NPM and p53. Lin et al (25) found NPM also interacted with the over-phosphorylated Rb protein. We found NPM colocalized with $\mathrm{Rb}$ at the nucleolar periphery. We provided evidence for further probing the detailed regulatory mechanism of NPM through interaction with $\mathrm{Rb}$.

Our research proved the expression changes of NPM in the differentiation of human liver tumor cells, as well as its colocalization and interaction with c-Fos, c-Myc, P53, Rb, $\mathrm{Bcl}-2$ and $\mathrm{Bcl}-\mathrm{xl}$. As a regulatory factor, NPM played pivotal roles in regulating liver tumor cell differentiation induced with HMBA, indicating NPM may be the potential target protein of HMBA. The location and expression changes of NPM significantly affected the proliferation and differentiation of liver tumor cells. NPM regulated cell differentiation through interacting with the proteins from oncogenes and tumor suppressor genes. Exploring the undiscovered roles of NPM is of great significance for revealing the regulation of human liver cell carcinogenesis and its reversal.

Collectively, the present study showed that NPM was overexpressed both in liver tumor cells and in liver cancer tissues; its expression level and location were altered during the HMBA-induced differentiation. NPM colocalized and interacted with c-Myc, c-Fos, P53 and Rb and the colocalizations were eliminated or translocated by HMBA treatment, indicating the functional roles of NPM during the differentiation of liver tumor cells. Further investigation on the molecular mechanism of NPM regulating cancer cell differentiation will help to reveal its detailed functional roles.

\section{Acknowledgements}

This study was supported by National Natural Science Foundation of China (Grant numbers: 81272245, 81272921 and 81201305), Natural Science Foundation of Fujian Province (Grant numbers: 2011J01256 and 2013J01359) and Joint Programme by Healthy Care System and Educational Department in Fujian Province (Grant number: WKJ-FJ-16).

\section{References}

1. Grisendi S, Mecucci C, Falini B and Pandolfi PP: Nucleophosmin and cancer. Nat Rev Cancer 6: 493-505, 2006.

2. Lindström MS: Elucidation of motifs in ribosomal protein $S 9$ that mediate its nucleolar localization and binding to NPM1/ nucleophosmin. PLoS One 7: e52476, 2012.

3. Lim MJ and Wang XW: Nucleophosmin and human cancer. Cancer Detect Prev 30: 481-490, 2006.

4. Tsui KH, Juang HH, Lee TH, Chang PL, Chen CL and Yung BY: Association of nucleophosmin/B23 with bladder cancer recurrence based on immunohistochemical assessment in clinical samples. Acta Pharmacol Sin 29: 364-370, 2008. 
5. Hsu CY and Yung BY: Over-expression of nucleophosmin/B23 decreases the susceptibility of human leukemia HL-60 cells to retinoic acid-induced differentiation and apoptosis. Int $\mathbf{J}$ Cancer 88: 392-400, 2000.

6. Li QF, Shi SL, Liu QR, Tang J, Song J and Liang Y: Anticancer effects of ginsenoside $\operatorname{Rg} 1$, cinnamic acid, and tanshinone IIA in osteosarcoma MG-63 cells: nuclear matrix downregulation and cytoplasmic trafficking of nucleophosmin. Int J Biochem Cell Biol 40: 1918-1929, 2008

7. Liang Y, Li QF, Zhang XY, Shi SL and Jing GJ: Differential expression of nuclear matrix proteins during the differentiation of human neuroblastoma SK-N-SH cells induced by retinoic acid. J Cell Biochem 106: 849-857, 2009.

8. Tang J, Niu JW, Xu DH, Li ZX, Li QF and Chen JA: Alteration of nuclear matrix-intermediate filament system and differential expression of nuclear matrix proteins during human hepatocarcinoma cell differentiation. World J Gastroenterol 13: 2791-2797, 2007.

9. Derenzini M, Sirri V, Trere D and Ochs RL: The quantity of nucleolar proteins nucleolin and protein B23 is related to cell doubling time in human cancer cells. Lab Invest 73: 497-502, 1995.

10. Michishita E, Kurahashi T, Suzuki T, et al: Changes in nuclear matrix proteins during the senescence-like phenomenon induced by 5-chlorodeoxyuridine in HeLa cells. Exp Gerontol 37 885-890, 2002.

11. Hsu CY and Yung BY: Down-regulation of nucleophosmin/B23 during retinoic acid-induced differentiation of human promyelocytic leukemia HL-60 cells. Oncogene 16: 915-923, 1998.

12. Dergunova NN, Bulycheva TI, Artemenko EG, et al: A major nucleolar protein B23 as a marker of proliferation activity of human peripheral lymphocytes. Immunol Lett 83: 67-72, 2002.

13. Subong EN, Shue MJ, Epstein JI, Briggman JV, Chan PK and Partin AW: Monoclonal antibody to prostate cancer nuclear matrix protein (PRO:4-216) recognizes nucleophosmin/B23. Prostate 39: 298-304, 1999.

14. Nozawa Y, Van Belzen N, Van der Made AC, Dinjens WN and Bosman FT: Expression of nucleophosmin/B23 in normal and neoplastic colorectal mucosa. J Pathol 178: 48-52, 1996.
15. Zhang Y: The ARF-B23 connection: implications for growth control and cancer treatment. Cell Cycle 3: 259-262, 2004.

16. Ulanet DB, Torbenson M, Dang CV, Casciola-Rosen L and Rosen A: Unique conformation of cancer autoantigen B23 in hepatoma: a mechanism for specificity in the autoimmune response. Proc Natl Acad Sci USA 100: 12361-12366, 2003.

17. You BJ, Huang IJ, Liu WH, Hung YB, Chang JH and Yung BY Decrease in nucleophosmin/B23 mRNA and telomerase activity during indomethacin-induced apoptosis of gastric KATO-III cancer cells. Naunyn Schmiedebergs Arch Pharmacol 360: 683-690, 1999.

18. Gjerset RA: DNA damage, p14ARF, nucleophosmin (NPM/B23), and cancer. J Mol Histol 37: 239-251, 2006.

19. Li Z, Boone D and Hann SR: Nucleophosmin interacts directly with c-Myc and controls c-Myc-induced hyperproliferation and transformation. Proc Natl Acad Sci USA 105: 18794-18799, 2008.

20. Yung BY: c-Myc-mediated expression of nucleophosmin/B23 decreases during retinoic acid-induced differentiation of human leukemia HL-60 cells. FEBS Lett 578: 211-216, 2004.

21. Arabi A, Rustum C, Hallberg E and Wright AP: Accumulation of c-Myc and proteasomes at the nucleoli of cells containing elevated c-Myc protein levels. J Cell Sci 116: 1707-1717, 2003.

22. Grandori C, Gomez-Roman N, Felton-Edkins ZA, et al: c-Myc binds to human ribosomal DNA and stimulates transcription of rRNA genes by RNA polymerase I. Nat Cell Biol 7: 311-318, 2005.

23. Maiguel DA, Jones L, Chakravarty D, Yang C and Carrier F: Nucleophosmin sets a threshold for p53 response to UV radiation. Mol Cell Biol 24: 3703-3711, 2004.

24. Colombo E, Marine JC, Danovi D, Falini B and Pelicci PG: Nucleophosmin regulates the stability and transcriptional activity of p53. Nat Cell Biol 4: 529-533, 2002.

25. Lin CY, Liang YC and Yung BY: Nucleophosmin/B23 regulates transcriptional activation of E2F1 via modulating the promoter binding of NF-kappaB,E2F1 and pRB. Cell Signal 18: 2041-2048, 2006. 